\title{
Sensitivity of low degree oscillations to the change in solar abundances
}

\author{
A. Zaatri ${ }^{1,2}$, J. Provost ${ }^{1}$, G. Berthomieu ${ }^{1}$, P. Morel $^{1}$, and T. Corbard ${ }^{1}$ \\ 1 Département Cassiopée, UMR CNRS 6202, Observatoire de la Côte d'Azur, BP 4229, 06304 Nice Cedex 4, France \\ e-mail: zaatri@obs-nice.fr \\ 2 Centre de Recherche en Astronomie, Astrophysique et Géophysique, BP 63, Route de l'Observatoire, Bouzaréah, 16340, Alger, \\ Algérie
}

Received 31 January 2007 / Accepted 11 April 2007

\begin{abstract}
Context. The most recent determination of the solar chemical composition, using a time-dependent, 3D hydrodynamical model of the solar atmosphere, exhibits a significant decrease of C, N, O abundances compared to their previous values. Solar models that use these new abundances are not consistent with helioseismological determinations of the sound speed profile, the surface helium abundance and the convection zone depth.

Aims. We investigate the effect of changes of solar abundances on low degree p-mode and g-mode characteristics which are strong constraints of the solar core. We consider particularly the increase of neon abundance in the new solar mixture in order to reduce the discrepancy between models using new abundances and helioseismology.

Methods. The observational determinations of solar frequencies from the GOLF instrument are used to test solar models computed with different chemical compositions. We consider in particular the normalized small frequency spacings in the low degree p-mode frequency range.

Results. Low-degree small frequency spacings are very sensitive to changes in the heavy-element abundances, notably neon. We show that by considering all the seismic constraints, including the small frequency spacings, a rather large increase of neon abundance by about $(0.5 \pm 0.05)$ dex can be a good solution to the discrepancy between solar models that use new abundances and low degree helioseismology, subject to adjusting slightly the solar age and the highest abundances. We also show that the change in solar abundances, notably neon, considerably affects g-mode frequencies, with relative frequency differences between the old and the new models higher than $1.5 \%$.
\end{abstract}

Key words. Sun: helioseismology - Sun: abundances - Sun: interior

\section{Introduction}

The precise measure of characteristics of the observed p-modes has been used to probe most of the layers inside the sun. For example, seismic sound speed and density determinations can be used to constrain the interior of the sun anywhere except at the surface and in the core. Helioseismology also constrains the surface helium abundance and the depth of the convection zone. However, the small number of p-modes (only low degree p-modes) able to reach the solar core is not sufficient to probe this region using inversion techniques. The solar core is crossed by thousands of $\mathrm{g}$-modes, able to bring much information from this region. The g-modes have not yet been unambiguously identified because of their evanescent nature through the convection zone and low amplitude at the surface but ongoing work is devoted to try to extract them for the existing long time series of SOHO data and to propose new observational and strategies to detect them.

New determinations of solar heavy element abundances using a 3D, NLTE analysis of the solar spectrum has been provided by Asplund et al. (2005; AGS). Previous 1D, LTE determinations are available (Grevesse \& Noels 1993 - GN; Grevesse \& Sauval 1998 - GS). Relative to GN abundances, the new AGS abundances are, among others, lower in C, N, O, Ne elements by respectively $0.16 \mathrm{dex}, 0.19 \mathrm{dex}, 0.21 \mathrm{dex}$ and $0.24 \mathrm{dex}$. Consequently, the new chemical determination gives a smaller metallicity $Z / X$ compared to the older ones. The new determination of solar elements is more accurate than the older one (Grevesse et al. 2005), but it has been shown that it leads to solar models that disagree with the helioseismological determinations of solar internal structure parameters (e.g. Turck-Chièze et al. 2004; Bahcall et al. 2005; Guzik et al. 2005).

In this paper we study the sensitivity of the solar core properties, through low degree p-modes and g-modes, to the change of solar mixture. The chemical solar abundances that we used are those of Grevesse \& Noels (1993), Grevesse \& Sauval (1998) and Asplund et al. (2005). The other mixtures that we chose include the solar abundances of Asplund et al. (2005) changing mainly the neon abundance. This set of solar mixtures allows us to study the influence of the neon abundance on small frequency separations and to test the possibility of improving the accordance between models that use new abundances and helioseismic observations (Antia \& Basu 2005; Bahcall et al. 2005). This is indeed possible because neon photospheric abundance cannot be determined directly due to the lack of suitable absorption lines in the solar spectrum induced by the noble gas nature of neon. The estimations of the solar $\mathrm{Ne}$ abundance are still controversial (Drake \& Tesla 2005; Young 2005; Schmelz 2005). Here we analyze how the models fit both the global constraints (seismic sound speed, surface helium abundance and convection zone depth) and the small frequency separations in the low degree p-mode frequency range. We use the determination of these 
Table 1. Global characteristics of the computed solar models. $A(\mathrm{Ne})$ is the neon abundance in dex, $(Z / X)_{\mathrm{S}}$ is the surface metallicity, $T_{\mathrm{c}}^{7}=T_{\mathrm{c}} \times$ $10^{-7}, T_{\mathrm{c}}$ the central temperature in Kelvin. $P_{0}$ is the characteristic period (in minutes) of low degree gravity modes. The different models are computed with the following solar abundances: M-GN: GN; M-GS: GS; M-GS*: GS with sulfur abundance of GN; M-AGS: AGS; M3, M4, M5, M6, M7: AGS with the indicated change of the neon abundance; M8: in addition to the change of neon in AGS, C, N, O, Mg and Si have been increased until the maximum of their error bars (see Asplund et al. 2005) and Ar by 0.40 dex.

\begin{tabular}{lllllll}
\hline \hline & $A(\mathrm{Ne})$ & $(Z / X)_{\mathrm{S}}$ & $Y_{\mathrm{S}}$ & $r_{\mathrm{ZC}}$ & $T_{\mathrm{c}}^{7}$ & $P_{0}$ \\
\hline M-GN & 8.08 & 0.0245 & 0.2437 & 0.7133 & 1.574 & 35.08 \\
M-GS & 8.08 & 0.0232 & 0.2462 & 0.7165 & 1.574 & 35.03 \\
M-GS & 8.08 & 0.0231 & 0.2429 & 0.7153 & 1.571 & 35.15 \\
M-AGS & 7.84 & 0.0166 & 0.2279 & 0.7292 & 1.549 & 35.68 \\
M3 & 8.10 & 0.0179 & 0.2329 & 0.7237 & 1.555 & 35.49 \\
M4 & 8.29 & 0.0192 & 0.2381 & 0.7181 & 1.559 & 35.29 \\
M5 & 8.35 & 0.0198 & 0.2402 & 0.7162 & 1.561 & 35.21 \\
M6 & 8.40 & 0.0203 & 0.2417 & 0.7144 & 1.563 & 35.15 \\
M7 & 8.47 & 0.0212 & 0.2445 & 0.7119 & 1.565 & 35.04 \\
M8 & 8.35 & 0.0213 & 0.2439 & 0.7142 & 1.566 & 35.05 \\
\hline
\end{tabular}

mode frequencies obtained from the GOLF experiment by Gelly et al. (2002) and more recently by Lazrek et al. (2007) who have corrected these frequencies for the solar cycle effect. The sensitivity of gravity modes to the new abundances have also been estimated. Preliminary results of this work have been presented by Zaatri et al. (2006).

\section{Solar models with new abundances}

We have computed solar models with different sets of heavy element abundances by using the stellar evolution code CESAM (Morel 1997). We use OPAL opacity tables ${ }^{1}$, calculated for each mixture, and Alexander and Ferguson opacity tables at low temperatures $(T<6000 \mathrm{~K})$. Nuclear reaction rates are from NACRE compilation (Angulo et al. 1999) and equation of state tables are those of OPAL (Iglesias \& Rogers 1991). We assume the convection treatment given by Canuto \& Mazitelli (1991). All the models include the microscopic diffusion of the chemical elements according to the Michaud \& Proffitt (1991) description. Models are calibrated for a solar age $t=4.6 \mathrm{Gyr}$ at the solar radius, the solar luminosity $\left(R_{\odot}=6.9599 \times 10^{10} \mathrm{~cm}, L_{\odot}=3.846 \times 10^{33} \mathrm{erg} / \mathrm{s}\right.$, Christensen-Dalsgaard et al. 1996) and the solar surface metallicity $Z / X$ of the various mixtures.

Table 1 summarizes the characteristics of the solar models at both the surface and the core and their chemical composition is given. The surface helium abundance and the location of the base of the convection zone $Y_{\mathrm{s}}$ and $r_{\mathrm{ZC}}$ are to be compared to their seismic determinations $Y \mathrm{~s}=0.2485 \pm 0.0034$, $r_{\mathrm{ZC}}=(0.7133 \pm 0.001) R_{\odot}$ by Basu \& Antia (2004). These authors have demonstrated that these seismic determinations are not sensitive to the change in solar abundances.

Figure 1 shows relative differences between seismic sound speed and the one determined from our different solar models. Figure 2 shows a comparison between $Y_{\mathrm{s}}$ and $r_{\mathrm{ZC}}$ values of the computed models and their seismic determinations. The worse concordance between the model using Asplund et al. abundances and the seismic model is shown by a relative difference in sound speed that peaks at $1.5 \%$ just below the convection zone. The surface helium abundance and the location of the base of the convection zone are also very far from their seismic values.

${ }^{1}$ http://www-pat.1lnl.gov/Research/OPAL/

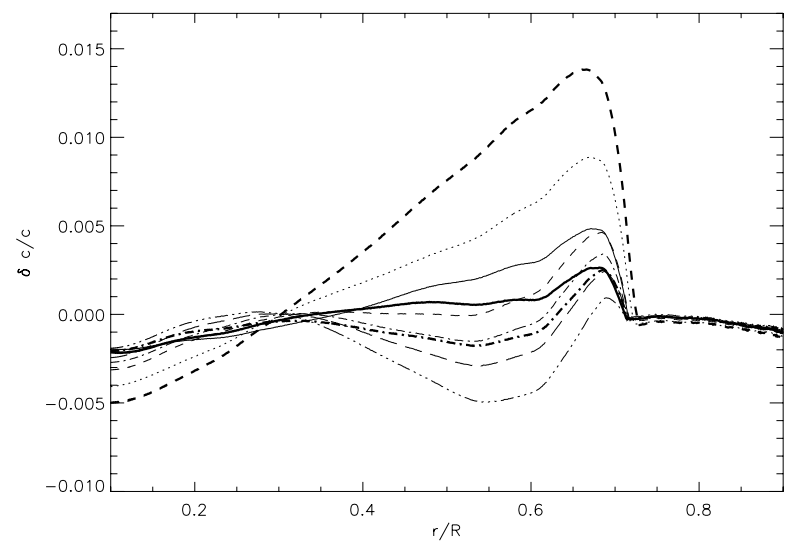

Fig. 1. Relative sound speed differences between the Sun and the models: M-GN (heavy full), M-GS (full), M-AGS (heavy dashed), M3 (dotted), M4 (short-dashed), M5 (dashed-dotted), M6 (long-dashed), M7 (dashed- $3 *$ dotted), M8 (heavy dashed-dotted).

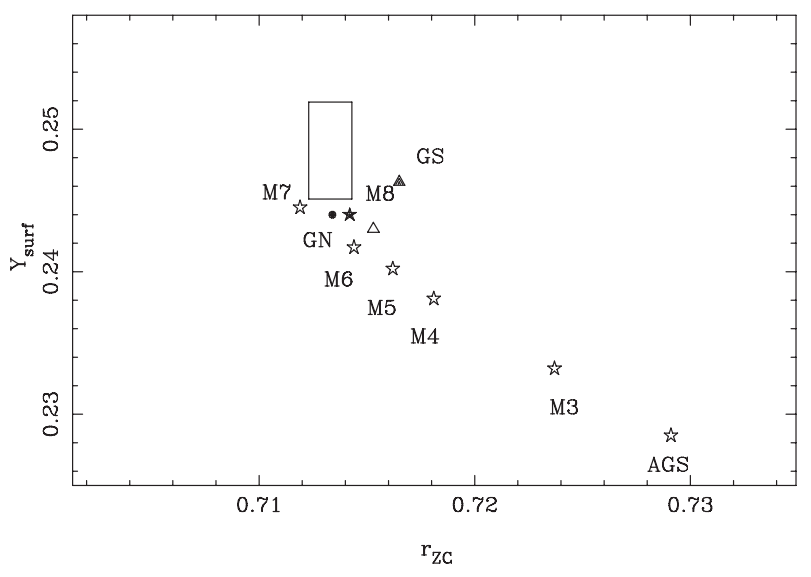

Fig. 2. Characteristics of the solar envelope, surface helium abundance $\left(Y_{\mathrm{S}}\right)$ and the location of the base of the convection zone $\left(r_{\mathrm{ZC}}\right)$, for the models: M-GN (filled circle), M-GS (filled triangle), M-GS* (open triangle), M-AGS (open star), M3 to M7 which is a set of models that use AGS mixture by varying its neon abundance (open star) and M8 (filled star). The box represents the seismic values with their errors (Basu \& Antia 2004).

Models M3, M4, M5, M6 and M7 give an idea of how large the neon abundance increase has to be in order to reduce this discrepancy. In all these models the neon has been pushed out of its error bar $( \pm 0.06 \mathrm{dex})$. Before looking for an optimal value of neon, we notice that the larger the neon abundance, the larger the surface helium abundance, the larger the convection zone depth and the higher the sound speed. The augmentation of neon in M4 makes the model's sound speed close to the seismic profile but keeps $Y_{\mathrm{s}}$ and $r_{\mathrm{ZC}}$ far from their seismic box, even if they become better than the M-AGS ones. A slightly higher increase of neon (e.g. M7) improves the surface envelope characteristics $\left(Y_{\mathrm{s}}, r_{\mathrm{ZC}}\right)$ but makes the sound speed much higher than the seismic one. Therefore, a compromise has to be found for the neon abundance value to satisfy $Y_{\mathrm{s}}, r_{\mathrm{ZC}}$ and sound speed constraints. We estimate the neon abundance to be $8.39 \pm 0.05$ dex, subject to some remaining differences between the models and the observation. First, the lowest values of this range lead to models that have $Y_{\mathrm{S}}$ and $r_{\mathrm{ZC}}$ values about $2 \sigma$ far from their seismic values. Second, its highest values infer models with relative differences between their sound speed profile and the seismic one that 
are just three times lower than the relative difference between M-AGS and seismic sound speed profiles at their peaks.

In order to see the influence of the other heavy elements abundances on the change of the considered model characteristics ( $Y_{\mathrm{S}}, r_{\mathrm{ZC}}$ and sound speed) we constructed the M8 model. This model has a neon abundance that is situated at the bottom of our given neon increase interval (8.35 dex) and has $\mathrm{C}, \mathrm{N}, \mathrm{O}$, $\mathrm{Si}$ and $\mathrm{Mg}$ abundances that are increased until the maximum of their error bars (see Asplund et al. 2005). The abundance of argon has also been increased by 0.4 dex, as this element is another noble gas of the solar mixture. We deduce that the sound speed of the M8 model does not change much compared to the model that has the same increase of neon (M5), except at the deeper layers of the sun. However, the values of $Y_{\mathrm{S}}$ and $r_{\mathrm{ZC}}$ become closer to their seismic values compared to those of the M5 model. This means that the increase of other heavy element abundances inside their error bars simultaneously improves the agreement between the three considered seismic determinations and those of the models. This can lead to a reduction of the supposed values of neon as they give good agreement in sound speeds to $(8.34 \pm 0.05)$ dex. For this range, relative differences between seismic and theoretical $Y_{\mathrm{S}}$ and $r_{\mathrm{ZC}}$ still have a maximum of $2 \sigma$.

For comparison we have considered the models M-GN and M-GS with the previous mixtures GN and GS. The model M-GN has a convective zone depth close to the seismic one but a too small surface helium abundance. On the contrary, model M-GS has a good surface helium content but a thinner convective zone. Since in Fig. 2, the position of the M-GS model does not follow the general trend of the other models, we have looked in more detail at the differences between the two mixtures. We noted that the sulfur abundance of GS mixture (7.33 dex) is larger than the GN value (7.21 dex), due to improved oscillator strengths (Biemont et al. 1993), and than the AGS value (7.14 dex). We computed a model M-GS* with the GS mixture but with GN sulfur abundance. It appears that such a variation of sulfur notably lowers the surface helium abundance of the model.

\section{Fit to the low-degree small frequency spacing constraints}

Small low degree frequency spacings are a well known diagnostic of the solar core. In order to compare our theoretical results to the observational ones, we use the latest results of Lazrek et al. (2007) and those of Gelly et al. (2002) in the measurement of low degree solar frequencies from the GOLF experiment. We examine the small frequency spacings $\delta v_{02}, \delta v_{13}$ and $\delta v_{01}$ which are combinations of acoustic modes penetrating differently towards the center and that are thus very sensitive to this region.

$$
\begin{aligned}
& \delta v_{02}(n)=v_{n, \ell=0}-v_{n-1, \ell=2}, \\
& \delta v_{13}(n)=v_{n, \ell=1}-v_{n-1, \ell=3}, \\
& \delta v_{01}(n)=2 v_{n, \ell=0}-\left(v_{n, \ell=1}+v_{n-1, \ell=1}\right) .
\end{aligned}
$$

However, the small spacings are slightly dependent on the solar atmosphere which is highly simplified in the solar models. Roxburgh \& Vorontsov (2003) have demonstrated that the ratio of the small to large separations of acoustic oscillations is essentially independent of the structure of the outer layers. We thus renormalize the small spacings by considering the ratios $\delta v_{02}(n) / \Delta v(n, \ell=1), \delta v_{13}(n) / \Delta v(n+1, \ell=0)$ and $\delta v_{01}(n) / \Delta v(n, \ell=1)$ where the large separation is given by:

$\Delta v(n, \ell)=v_{n, \ell}-v_{n-1, \ell}$.
We then compute both for our models and for the observations the mean of these renormalized small frequency spacings $\overline{\delta v_{02}}$, $\overline{\delta v_{13}}$ and $\overline{\delta v_{01}}$ for radial orders from 16 to 24 . This corresponds to a frequency range about $2500-3600 \mu \mathrm{Hz}$. The low limit of this range ensures that the behavior of the frequency is almost asymptotic and the high limit corresponds to observed modes with very high accuracy. For higher frequencies, the accuracy decreases rapidly. Figure 3 gives both renormalized and non renormalized mean small spacings for the models of Table 1 and for the observations. The dimensions of the symbols in the upper panel of Fig. 3 reflect the uncertainty on the plotted quantities corresponding to an uncertainty of $0.01 \mu \mathrm{Hz}$ for the numerical frequencies. It shows that the renormalization gives results closer to the observations because it eliminates the differences between surface properties of the models and the sun. The remaining discrepancies are due to differences in the structure of the deep solar interior.

As expected, Fig. 3 shows that the small frequency spacings of the M-AGS model are far from the observations, contrary to those of the M-GN and M-GS models. We note that the M-GS model is closer to the observations than the M-GN model, contrary to the M-GS* model. The small frequency spacings of the models that use an AGS mixture with different values of the neon abundance decrease as the neon increases in almost a regular way. The model M7, which uses the highest value of neon abundance in our considered set of models, is shown to have the best agreement between its small spacings and the observations. However, this model has a much higher sound speed than the seismic one (see Fig. 1), which makes it a less acceptable model. We also show that a slight increase of some other heavy elements has an effect on the change of small frequency spacings as well. We find that for the model M8 the three considered renormalized small spacings are closer to their observational determinations than those of the model M5.

However the small spacings are also sensitive to the solar age, due to the change in time of the mean molecular weight in the nuclear core. For example, Morel et al. (1997) showed that an increase in age of $100 \mathrm{Myr}$ gives a decrease of $0.1 \mu \mathrm{Hz}$ of both $\overline{\delta v_{02}}$ and $\overline{\delta v_{13}}$, with a small relative increase of sound speed of around $10^{-3}$ and a slight increase of the thickness of the convection zone of $0.002 R_{\odot}$ and no noticeable change of the surface helium abundance. We added, in Fig. 3 a GN model which is calibrated at 4.65 Gyr to see the influence of the solar age on small frequency spacings.

After showing the influence of solar abundances on low degree small frequency spacings, we still believe that in order to resolve the new abundances, a compromise between the neon abundance, the small frequency spacings and the constraints of the preceding paragraph can be reached by slightly adjusting some heavy elements inside their error bars and the age of the sun. Our suggested value of neon $(8.34 \pm 0.05)$ is confirmed by considering the low degree small frequency spacing constraint.

\section{Gravity modes}

Adiabatic frequencies of the models have also been computed for the range from 100 to $800 \mu \mathrm{Hz}$ and for low degrees $(0<$ $\ell<6$ ) including both $g$-modes and mixed modes. The periods of low frequency gravity modes are proportional to the characteristic period $P_{0}$ which is given in Table $1\left(P_{0}=2 \pi^{2} / \int_{0}^{r \mathrm{ZC}}(N / r) \mathrm{d} r\right.$, where $N$ is the Brunt-Väissälä frequency). The lowest $P_{0}$ difference between M-GN and the other models is obtained for M8, leading to the closest g-mode frequencies. The frequency 

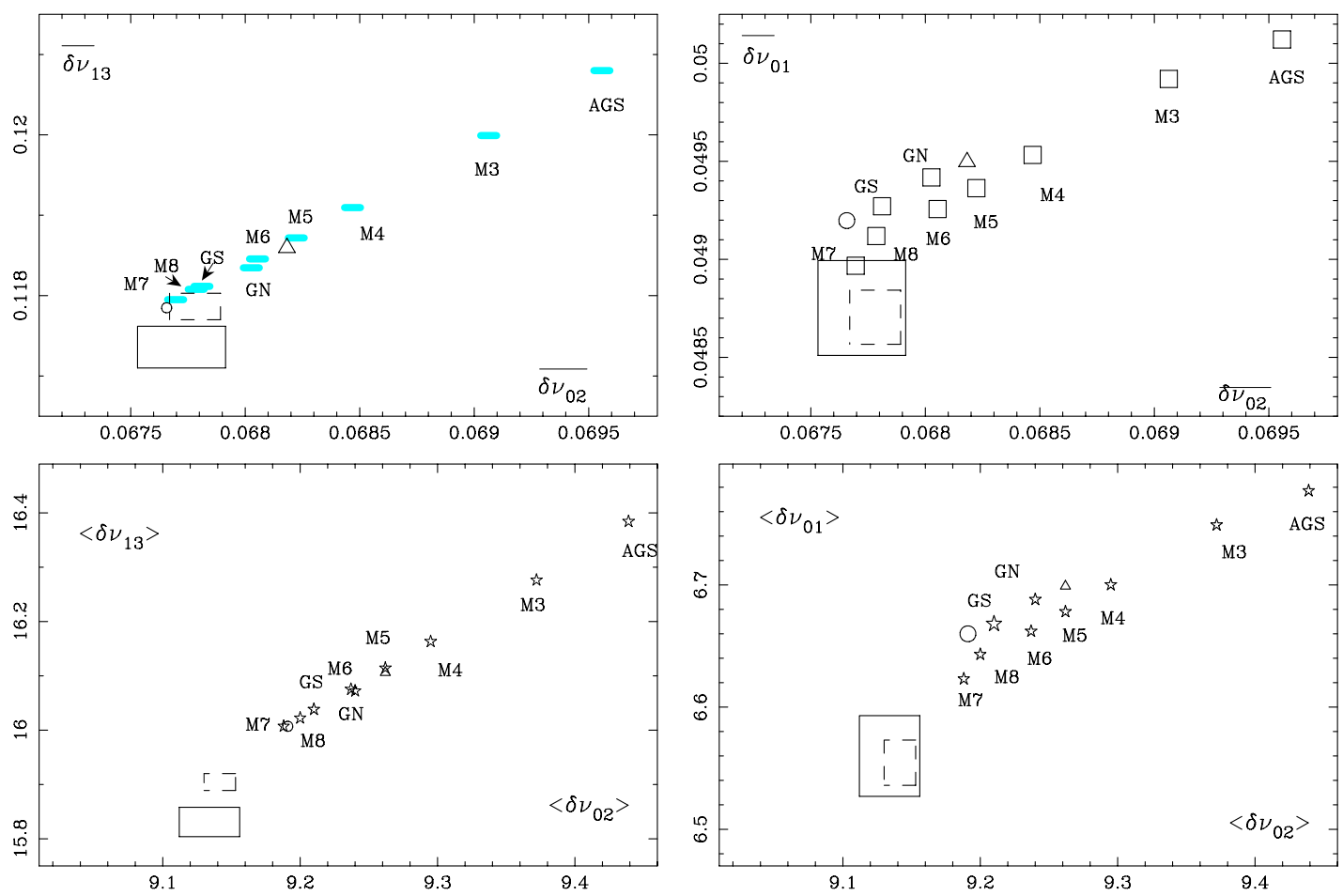

Fig. 3. Upper panel left: renormalized mean frequency small spacing $\overline{\delta v}_{13}$ as a function of the renormalized mean frequency small spacing $\overline{\delta v}_{02}$ for the different models compared to GOLF observations (full box Gelly et al. 2002; dashed box Lazrek et al. 2007). Upper panel right: same for renormalized mean frequency small spacing $\overline{\delta v}_{01}$ as a function of the renormalized mean frequency small spacing $\overline{\delta v}_{02}$. Lower panel: for comparison the same quantities are given before renormalization (in $\mu \mathrm{Hz}$ ), namely $\left\langle\delta v_{13}\right\rangle$ and $\left\langle\delta v_{01}\right\rangle$ as a function of $\left\langle\delta v_{02}\right\rangle$. The small circle corresponds to a M-GN model calibrated at $4.65 \mathrm{Gyr}$, the open triangles correspond to M-GS* model.

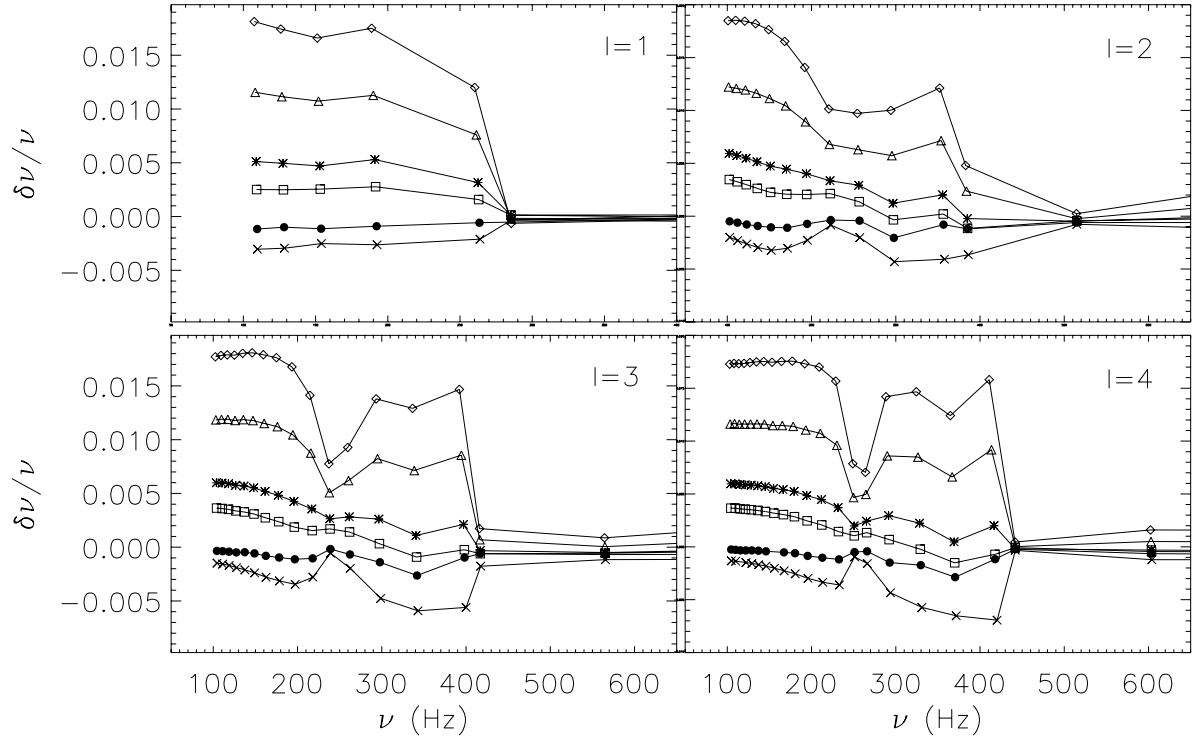

Fig. 4. Relative frequency differences between the gravity modes and the first low frequency p-modes of M-GN and M-AGS $(\diamond)$, M3 $(\triangle)$, M4(*), M5 (), M7(×), M8(•). differences between the M-GN and the other models are given in Fig. 4. We note that the g-modes are more influenced by the change of abundances than the low frequency p-modes. As expected the value of $\delta v / v$ at very low frequency is close to its asymptotic value $\delta P_{0} / P_{0}$. The biggest frequency difference is obtained for M-AGS for which low g-mode frequencies are $1.5 \%$ lower. This difference has a minimum for all the models around $250 \mu \mathrm{Hz}$. It has been shown that the g-modes around this frequency have a mixed character (Provost et al. 2000) and are sensitive to both the sound speed and the Brunt-Väissälä frequency variations. Their frequencies do not depend much on any change in the models. The lowest difference in the frequencies compared to M-GN is obtained for M8 which is expected since they have very similar structure parameters.

\section{Discussion and conclusion}

We study the sensitivity of low degree frequency spacings to the change on solar heavy element abundances. We constructed several solar models with different solar mixtures. The spacings are considered as helioseismic constraints of the solar core as they are very sensitive to this deep region. Therefore, they are used to test the reliability of the solar models in addition to the envelope constraints (convection zone depth and helium surface 
abundance) and the sound speed profile. Surface effects have been removed from these spacings by using a renormalization prescribed by Roxburgh \& Vorontsov (2003). Their observational values have been taken from the recent GOLF measurements (Lazrek et al. 2007; Gelly et al. 2002).

We found that low degree small frequency spacings are very sensitive to the metallicity of the models. The mean spacings of a model using Asplund et al. (2005) abundances are much higher than the ones of a model using Grevesse \& Noels (1993) values and are incompatible with those determined from the GOLF observations. Similar results were found by Basu et al. (2007) by comparing BISON observations of low degree solar oscillations with models using different abundances and numerical codes. They conclude that low surface metallicity models can be ruled out. These two studies strengthen the fact that new solar abundances lead to solar models which disagree with helioseismology measurement in the core as well as in the other regions of the solar interior.

We confirm, as was also mentioned by several authors, that the sound speed profile, convection zone depth and helium surface abundance of a model using the revised abundances are also far from their helioseismic determinations, unlike the ones of a model using the old abundances.

In addition to these two main models we constructed five other models that use new solar abundances with a significant change of the neon abundance. This has been done following the Antia \& Basu (2005) suggestion to resolve the new abundances. We found that the small spacings are very sensitive to the neon abundance value and decrease almost regularly when the neon increases. The discrepancy between models and observations is reduced simultaneously for the small frequency spacings and the other constraints when the neon abundance is considerably increased. However, the neon abundance that gives the best agreement between the models and the helioseismic determinations is hard to reach as it is a compromise solution between all these quantities. For instance, a model using a neon value increased by 0.45 dex (M4) makes its sound speed very close to the seismic sound speed but keeps its envelope characteristics (convection zone depth and helium surface abundance) far from their observational values. A model using a neon abundance increased by 0.63 dex (M7) has the opposite effect.

As expected, the search for the neon abundance value that gives a good agreement between models using new abundances and the seismic constraints including the small frequency spacings becomes easier if $\mathrm{C}, \mathrm{N}, \mathrm{O}, \mathrm{Si}, \mathrm{Mg}$ and $\mathrm{Ar}$ abundances are also slightly increased. Other elements may also play a significant role. We show for example that an increase of sulfur abundance, as is the case for the GS mixture, noticeably increases the surface helium abundance and lowers the small frequency spacings.Also, the solar age is a crucial feature in the determination of low-degree frequencies. Indeed, we tested a model using old abundances with an age $50 \mathrm{Myr}$ older than the age we have used to compute all the models (4.6 Gyr) and found that this change brings the spacings closer to the observations.

In conclusion, we show that, if the new solar mixture of Asplund et al. (2005) is confirmed, an increase of the neon abundance by $(0.5 \pm 0.05)$ dex can resolve the current disagreement caused by this mixture, subject to adjusting slightly the highest heavy-element abundances and the age of the sun in order to satisfy all the seismic constraints, notably the low-degree small frequency spacings. Our estimated increase of neon is slightly higher than that of Bahcall et al. (2005), which is $0.45 \pm 0.05$. However, in this work only low degree modes are used, while the higher degree modes also provide independent constraints. Also our model, which is the closest to the observations, has a surface metallicity content $Z$ smaller than the value determined by Antia \& Basu (2006) from higher degree modes using the dimensionless sound speed derivative in the solar convection zone.

Our last investigation in this work has been the calculation of g-mode frequencies since the detection of g-modes is one of the current challenges of solar observers. As expected, the solar model using new abundances has the highest frequency differences to the model using old abundances, which go up to $4 \mu \mathrm{Hz}$. We show that modes with frequencies around $250 \mu \mathrm{Hz}$ and degrees larger than 2 are less sensitive modes to the change in the abundances, with differences less than $2 \mu \mathrm{Hz}$.

Acknowledgements. We thank B. Pichon for his technical help, D. R. Alexander for giving us low temperature opacity tables for the revised mixture and the OPAL group for their online opacity tables code. We are grateful to G. Grec and M. Lazrek for communicating their results in advance of publication and to H. M. Antia for his constructive remarks. We also thank the "Programme PluriFormations Astérosismologie" from OCA for the financial support.

\section{References}

Antia, H. M., \& Basu, S. 2005, ApJ, 620, L129

Antia, H. M., \& Basu, S. 2006, ApJ, 644, 1292

Angulo, C., Arnould, M., Rayet, M., and the NACRE collaboration 1999, Nucl. Phys. A, 656, 1

Asplund, M., Grevesse, N., \& Sauval, A. J. 2005, Cosmic Abundances as Records of Stellar Evolution and Nucleosynthesis, ASP Conf. Ser., 336, 25 Alexander, D. R., \& Ferguson, J. W. 1994, ApJ, 437, 879A

Bahcall, J. N., Basu, S., \& Serenelli, A. M. 2005, ApJ, 631, 1281

Basu, S., \& Antia, H. M. 2004, ApJ, 606, L85

Basu, S., Chaplin, W. J., Elsworth, Y., New, R., Serenelli, A. M., \& Verner, G. A. 2007, ApJ, 655, 660

Biemont, E., Quinet, P., \& Zeippen, C. J. 1993, A\&AS, 102, 435

Canuto, V. M., \& Mazitelli, I. 1991, ApJ, 370, 295

Christensen-Dalsgaard, J., et al. 1996, Science, 272, 1286

Drake, J. J., \& Testa, P. 2005, Nature, 436, 525

Gelly, B., et al. 2002, A\&A, 394, 285

Gough, D. O. 1991, in Progress of Seismology of the Sun and Stars, ed. Y. Osaki, \& Shibahashi (Springer Verlag), 283

Grevesse, N., \& Noels, A. 1993, in Origin and Evolution of the elements, ed. Prantzos, Vangioni-Flam and Cassé (Cambridge Univ. press), 15

Grevesse, N., \& Sauval, A. G. 1998, Space Sci. Rev., 85(1/2), 161

Grevesse, N., Asplund, M., \& Sauval, A. G. 2005, in Element Stratification in Stars: 40 Years of Atomic Diffusion, ed. G. Alecion, O. Richard, \& S. Vauclair, EAS Publ. Ser., 17, 21

Guzik, J. A., Scott Watson, L., \& Cox, A. N. 2005, ApJ, 627, 1049

Iglesias, C. A., \& Rogers, F. J. 1991, ApJ, 371, 408

Lazrek, M., Grec, G., Fossat, E., \& Renaud, C. 2006, A\&A, submitted

Morel, P. 1997, A\&AS, 124, 597

Proffitt, C. R., \& Michaud, G. 1991, ApJ, 371, 584

Provost, J., Berthomieu, G., \& Morel, P. 2000, A\&A, 353, 775

Roxburgh, I. W., \& Vorontsov, S. V. 2003, A\&A, 411, 215

Schmelz, J. T., Nasraoui, K., Roames, J. K., Lippner, L. A., \& Garst, J. W. 2005, ApJ, 634, 197

Turck-Chièze, S., Couvidat, L., et al. 2004, Phys. Rev. Lett., 93, 21

Young, P. R. 2005, A\&A, 444, 45

Zaatri, A., Provost, J., Berthomieu, G., Morel, P., \& Corbard, T. 2006, in Beyond the spherical Sun, ed. K. Fletcher, \& M. Thompson, ESA SP-624, 59 